Communications in Physics, Vol. 24, No. 3S2 (2014), pp. 151-156

DOI:10.15625/0868-3166/24/3S2/5011

\title{
STUDY OF HANOI AND HOCHIMINH STOCK EXCHANGE BY ECONOPHYSICS METHODS
}

\author{
CHU THUY ANH, DAO HONG LIEN, NGUYEN TRI LAN AND NGUYEN AI VIET \\ Institute of Physics, Vietnam Academy of Science and Technology \\ E-mail: ctanh@iop.vast.ac.vn
}

Received 20 June 2014

Accepted for publication 20 August 2014

\begin{abstract}
The econophysics methods are used to study the financial fluctuation of Hanoi Stock Exchange (HNX) and Hochiminh Stock Exchange (HSX) in comparison to Dow John Industrial Average's one. The study has been made both on panic period (2007-2008) and on normal time of theses stocks. The results point out that for the period under consideration, the distribution of returns tends to be Student distribution. There exists an analogy between nonequilibrium phase transitions and financial market movement. Thus it is hypothesized that financial markets undergo self-organizing when the external volatility perception rises above some critical value, the distribution of signs of returns is to be similar to a second order phase transition. The results obtained by econophysics method have quite good agreement with the ones obtained by other economy analyzation methods.
\end{abstract}

Keywords: econophysics, phase transition.

\section{INTRODUCTION}

Econophysics, the name itself somehow explains the concept. It was the first time in 1995, the merging of the words "economics" and "physics" was introduced at the conference Dynamics of Complex Systems, which was held in Kolkata as a satellite meeting to the STATPHYS-19 [1]. Since 1991 [2], the pioneering work in the modern stream of economical physics was initiated. It is an interdisciplinary research field applying methods of statistical physics to problems in economics and finance. Econophysics does not literally apply the laws of physics to humans, but uses physical methods to study statistical properties of complex economic systems consisting of a large number of humans. Based on fundamental statistical mechanical models, they attempted to construct from simple rules complex behavior that could mimic the market and explain the price formation mechanism [3].

In physics, the concept of subordination can be found in the construction of non-Shannon entropies, in the limit of the continuos-time random walk, interface growth models and other statistical mechanical problems [4-6]. The mathematical-physical idea of subordination is that if the stochastic process is analyzed at the correct reference frame, it will always look like a simple Gaussian diffusion. But since we are dealing with stochastic processes, the reference frame is moving randomly as well; just enough for the actual process in observation to be described by

(C)2014 Vietnam Academy of Science and Technology 
some special cases of Gaussian distribution, such as Student one.

In finance and economy, we can not run experiments, we simply have one single unique realization of history. With the provided database, we wish to understand more about the underlying dynamics of markets and some undocumented properties. In this paper, we will see that there exists a spontaneous phase transition at the onset of panic.

\section{VOLATILITY AND DISTRIBUTION OF RETURNS}

In finance, return (also known as rate of return (ROR), or return on investment (ROI)) is the ratio of money gained or lost (whether realized or unrealized) on an investment relative to the amount of money invested [7].

For the purpose of the ongoing work, the only single period cases were considered. If the initial value of an investment is $V_{i}$, final value of that investment after time $\tau$ is $V_{f}$, then return is

$$
r=\frac{V_{f}-V_{i}}{V_{i}}
$$

Or it could be written

$$
r=\ln \left(\frac{V_{f}}{V_{i}}\right)=\ln \left(V_{f}\right)-\ln \left(V_{i}\right)
$$

The study has been made on database of Hanoi Stock Exchange and Hochiminh Stock Exchange, so the value of an investment is the price of a financial asset. Let $p_{t}$ be the price of a financial asset at time $t$, the return over a period of time $\tau$ is

$$
r_{t}=\frac{p_{t+\tau}-p_{t}}{p_{t}} \sim \ln \left(p_{t+\tau}\right)-\ln \left(p_{t}\right)
$$

The volatility of returns is plotted based on HNX database in the period of 10/2006 - 2/2011. The returns value varies around 0 , in normal times, shows that the market is in stable status, and varies stronger in panic times (2007-2008), express the unstable status of the market.

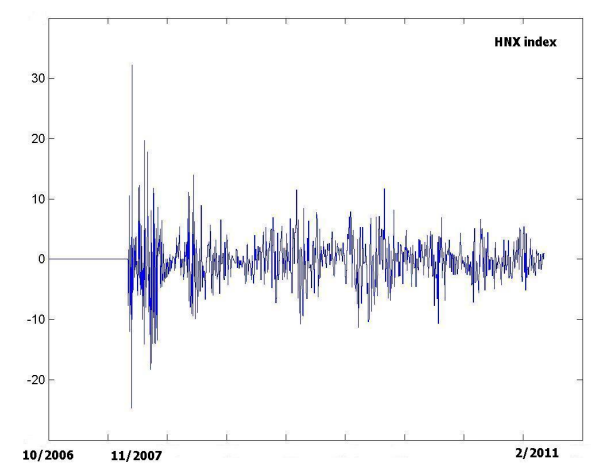

Fig. 1. Volatility of HNX's returns

The Dow John Industrial Average index (DJIA index) is also plotted for the period of 2006 2011. It's easy to see that the volatility tends to cluster, there are periods of high volatility followed by quieter periods. 


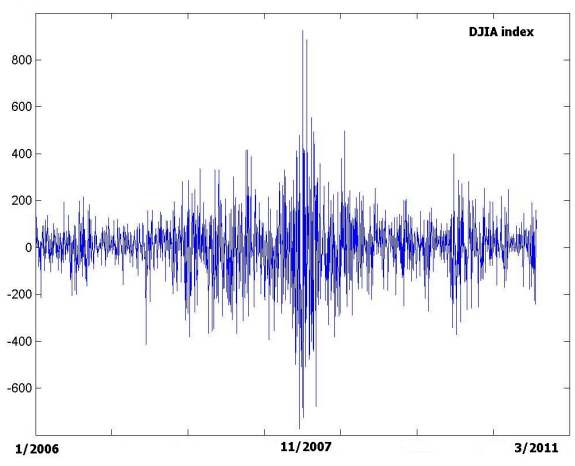

Fig. 2. Volatility of DJIA's returns

In comparing with DJIA volatility, HNX index varies with the same rhythm, it expresses the deep influence of global market fluctuation to inland market. It could also be remarked that there is some missing information for the beginning of the market. With the database provided by Hanoi Stock Exchange (HASTC), the share prices had absolutely no variation since the opening of the stock market until late 2007, but historically, we know that the HNX market was not so calm at the beginning.

In the graph below is the $\log$ (frequency) of the returns of HNX index from 10/2006 to $2 / 2011$, it's easy to see that it follows the Student distribution with sharp pick.

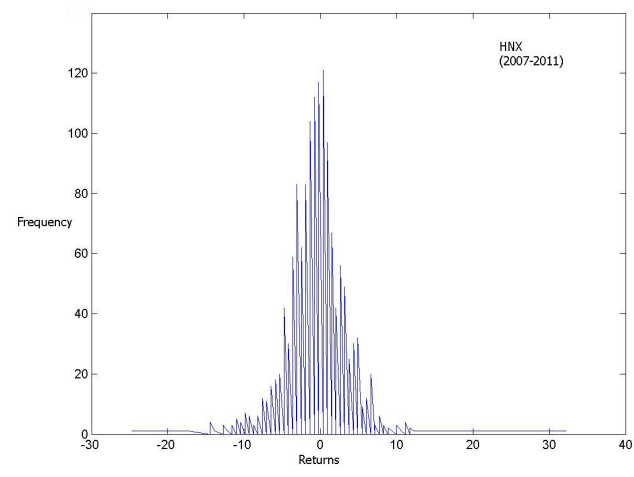

Fig. 3. $\log ($ frequency) of the returns of HNX index from $10 / 2006$ to $2 / 2011$

It has the max when the value of returns is around 0 , means no gain no lost, corresponds to the stable status of the market. So we can somehow say that the market returns now to it normal status after the financial crisis 2007-2008. But the returns tends to distribute more at negative values than at positive ones, while the $\log$ (frequency) of the returns of DJIA index in the same time (2006 - 2011) has a standard Student distribution. 


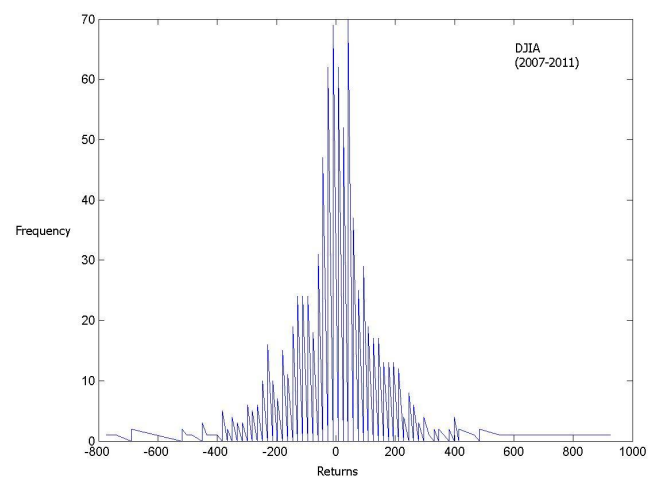

Fig. 4. $\log$ (frequency) of the returns of DJIA index from 2006 to $3 / 2011$

\section{PHASE TRANSITION MODEL}

Signs of return specifies the statistical features of runs which is defined as a sequence of consecutive gain/loss (rise/fall) stock returns [8].

With $u p$ the number of stocks that have positive returns over a given interval, and down the number of stocks that have negative moves on that same interval (for example a day), signs of returns

$$
s=\frac{u p-\text { down }}{u p+\text { down }}
$$

If $s=0$ we are in a disordered state, if $s \neq 0$ we are in an ordered state. So from the physical point of view, $s$ could be called the order parameter. It is a macroscopic parameter that tells whether there is order in the system or not. In physics, in particular in the field of non-equilibrium thermodynamic and synergetic [9], the concept of the order parameter is often used to describe systems that exhibit spontaneous self-organization. To illustrate the concept, let have a look at an example which should be familiar and intuitive to most, namely magnetism.

In a ferromagnetic system, the total magnetic moment depends on the orientation of the individual magnetic spins comprising the system. It is proportional to the quantity

$$
M=\frac{m_{u p}-m_{\text {down }}}{m_{u p}+m_{\text {down }}}
$$

where $m_{u p}$ and $m_{\text {down }}$ denote the number of spins lined up and down respectively. The distribution of possible outcome of this macroscopic quantity is given by

$$
P(m)=N e^{F(m, T)},
$$

where $T$ is the temperature, $N$ is a normalization of factor and $F$ is the free energy of the system. The temperature is an important parameter in this system because as we shall see, depending on the value of $T$, the magnetic system will either be in an ordered or disordered state. The free energy can be express as 


$$
F=\alpha\left(T-T_{c}\right) m^{2}+\beta m^{4}
$$

where $T_{c}$ is the critical temperature.

Potential $V(m)=-F(m)$ can be illustrated

\section{Potential Well}
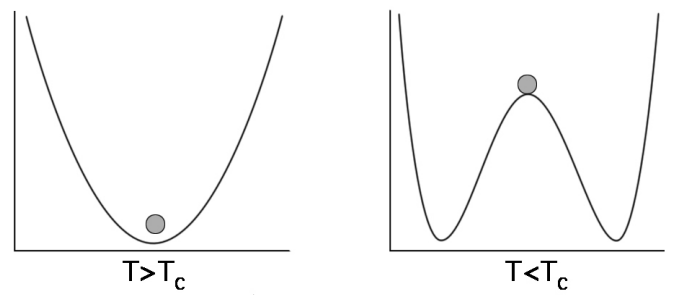

Fig. 5. Potential

It is easy to see that if $T>T_{c}$, the only minimum is the trivial one at $m=0$, and for $T<T_{c}$, there are two real roots appearing, yielding non-zero values of $m$. The probability distribution of the system in the disordered state will be a unimodal one, while the probability distribution of $m$ in the ordered state will be bimodal. As $T$ passes from above to below $T_{c}$, or vice-versa, there is clearly a phase transition: the state of the system is drastically altered. In this type of symmetric system, the phase transition if referred to as a second order one.

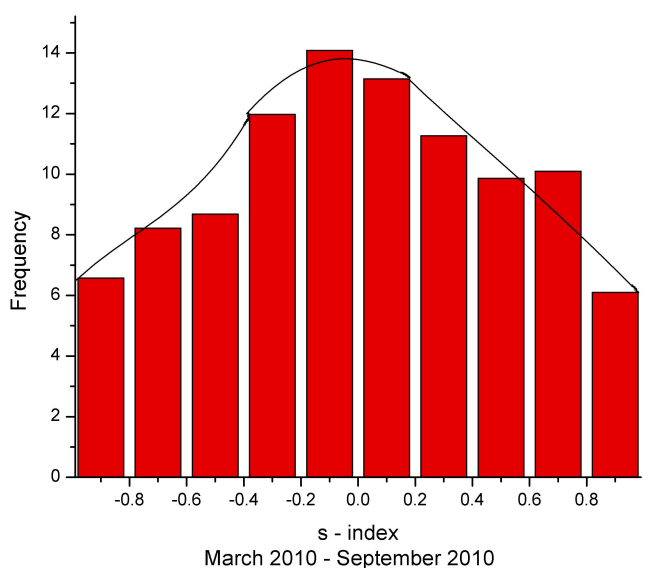

Fig. 6. s-index March to September 2010

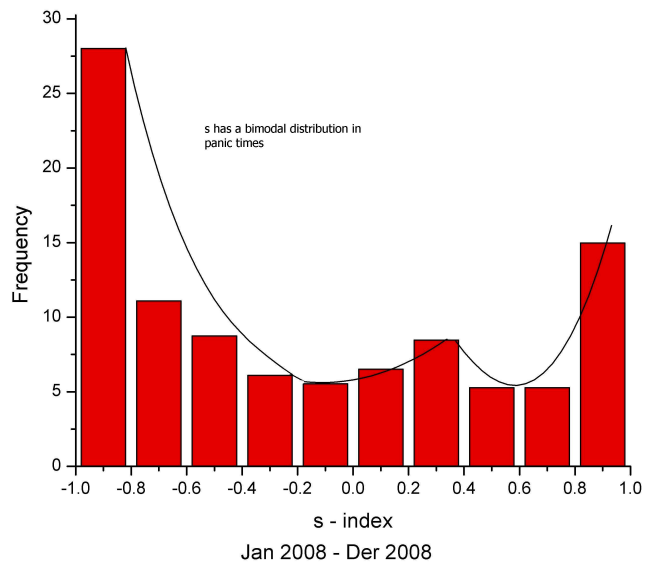

Fig. 7. s-index January to December 2008

The frequency of signs of returns has been plotted based on the data of HOSE for two period: January to December 2008 and March to September 2010. The studied period has been chosen based on the status of the market.

It is evidence that in the normal time (2010) frequency has a unimodal distribution and in the panic time (2008) it has a bimodal distribution. May be remarked here that there were a phase 
transition inside the market in between normal time and panic period. The distribution of signs of return in panic time is asymmetry, due to the chosen time. As we all know the panic time of Vietnamese stock market did not finish in December 2008, but due to the missing information from the stocks, we can not make our study for a longer period.

It's found that there is an analogy between the transition of the market and the phase transition in a ferromagnetic system as presented above.

\section{CONCLUSION}

Our finding shows that the general shape of the returns distribution respects well Student distribution. If the chosen period long enough, it should tend to Gaussian distribution [11]. Due to the missing data base of the stock market, we can not study VNI for a longer period. But somehow, our results based on physical point of view have quite good agreement with the one of economy analyzation. The comparison between VNI returns and DJIA returns shows that global market has important influences on our market.

We find also that there is a self-organization in financial market as well as in the phase transition. The appearance of critical points expresses some important fluctuations inside the system in both two cases, ferromagnetic one or economic one.

Although econophysics is a new branch of physics, but the "invasion" of physicists into economics and finance at the turn of the millennium is a fascinating phenomenon [10]; they draw the economic picture by the singular way. In this paper, we've just started to sketch some traits.

The affecting factors of the market has not been analyzed yet. In next presentations, we will define a kind of critical temperature for financial market and its self-organization process.

Part of this work has been supported by the Nafosted Grant No 103.06-2011.51.

\section{REFERENCES}

[1] A. Carbone, G. Kaniadakis, and A.M. Scarfone Physica A 382 (2007) 9-15.

[2] R. N. Mantegna, Physica A 179 (1991) 232.

[3] D. Challet and Y.-C. Zhang, Physica A 246 (1997) 407.

[4] C. Beck and E. G. D. Cohen, Physica A 322 (2003) 267.

[5] R. Failla, P. Grigolini, M. Ignaccolo, and A. Schwettmann, Phys. Rev. E 70 (2004) 010101.

[6] I. M. Sokolov, Phys. Rev. E 63, 011104 (2001).

[7] Stephen A. Ross, Randolph W. Westerfield, Jeffrey Jaffe, Coperate Finance, McGraw-Hill Higher Education(2010).

[8] Honggang Li and Yan Gao, New Economic Windows, Part I, 59-66, 2006.

[9] H. Haken, Synergetics: an introduction, Springer, 1977.

[10] Victor M. Yakovenko, J. Barkley Rosser, Jr, Rev. Mod. Phys. 81 (2009) 1703.

[11] Chu Thuy Anh, Do Hong Lien, and Nguyen Ai Viet, Comm. Phys., 23(2) (2013) 185. 